\title{
Atomic Force Microscopy in Food Research
}

\author{
A. Patrick Gunning \\ Institute of Food Research, Norwich Research Park, Colney, Norwich NR4 7UA
}

General examples the use of AFM in the study of biological systems in foods will be given. This talk will highlight the impact that structure has on virtually ever aspect of food, from perceived quality and desirability, which are largely determined by appearance and texture, through to the role that it plays in the release of nutrients and the safety of the food. Examples will be shown which encompass studies on biological systems with size ranges from the relatively large-scale structures seen at cellular levels, down to the interactions that occur at molecular and even submolecular scales that determine such functionality.

Approximately half of the foods that we eat are plant based, and in order to understand the functionality of such foods we naturally turn to microscopy as the principle means of revealing the structure of the plant tissue. Traditionally light microscopy has been used to visualise tissue microstructure (i.e. events at the cellular level), but in the search for ever more detailed understanding of these highly complex systems we require ever more detailed images. In microscopy terms put simply this means greater magnification, and until recently the only means of looking into the ultrastructure of plant tissues was the electron microscope. Good though it is, the electron microscope places quite severe restraints on the imaging environment, which are incompatible with the normal the physiological environment of plants and indeed food material in general. The advent of atomic force microscopy (AFM) has changed this (1). AFM has endowed us with the ability to examine plant tissue in a physiologically relevant environment and with a magnification range which spans the range of cells (ie. light microscopy) to single molecules (electron microscopy). Moreover it generates image contrast by a radically different mechanism to the traditional microscopies, which among other advantages eliminates the need for staining of the tissue, and so has great potential for new discovery. The image shown in figure 1 is a section of wheat endosperm which has received no other treatment than embedding in non-penetrating epoxy and cutting and was imaged in air with an AFM.

Image contrast is determined by a combination of surface topography and relative elastic modulus of the material. As can be seen this easily reveals all of the constituent organelles within the tissue. Why do we care what they look like? In order to understand factors such as the release of nutrients from plant tissue an understanding ultrastructure is crucial. For example the detailed nature of the cell walls in plant tissue strongly influence its behaviour upon both cooking and digestion, and example AFM images of different plant cell walls in their fully hydrated state will be shown which reveal clear differences attributable to the botanical origin that can be related to their functionality. The main source of energy from plant foods is derived from the breakdown of starches, and the contribution that AFM investigations into the ultrastructure of starch granules and its implications for subsequent enzymatic digestion will be illustrated $(2,3)$. Still at the cellular level, the role that food structure plays in preventing, (or in some case enhancing) spoilage by bacterial infection will be illustrated through studies on biofilm formation of Pseudomonas putida at oil-water interfaces. 
Many factors which dictate the functionality of foods occur not at the cellular level but at the molecular level. Examples of how AFM imaging can be brought to bear on this "nano-realm" will be given through studies ranging from the molecular interactions responsible for the stability of food emulsions, to those which allow the formation of food gels (figure 2) through to how AFM visualisation of population heterogeneity of plant polysaccharides has explained their functionality as texture enhancers. Furthermore, AFM visualisation of the effects of temperature on the aggregation of food proteins which dictate both their physical functionality and allergenicity will be discussed.

resolution.

Finally a unique feature of the AFM which sets it apart from any other form of microscopy is the ability to directly measure force interactions between food components as small as single molecules. This ability will be demonstrated through very recent studies (4) on the interaction between pairs of emulsion droplets (figure 3).

1. G. Binnig, C.F. Quate and Ch. Gerber, Phys. Rev. Letts. (1986) 930.

2. M.J. Ridout MJ, A.P. Gunning and M.L. Parker ML, et al. Carbohyd. Polym. (2002) 123.

3. T. Giardina, A.P. Gunning, and N. Juge et al., J. Mol. Biol. (2001) 1149.

4. A.P. Gunning, A.R. Mackie and P.J. Wilde et al. Langmuir (2004) 116.

5. This work was funded through the BBSRC by its core strategic research grant to IFR.

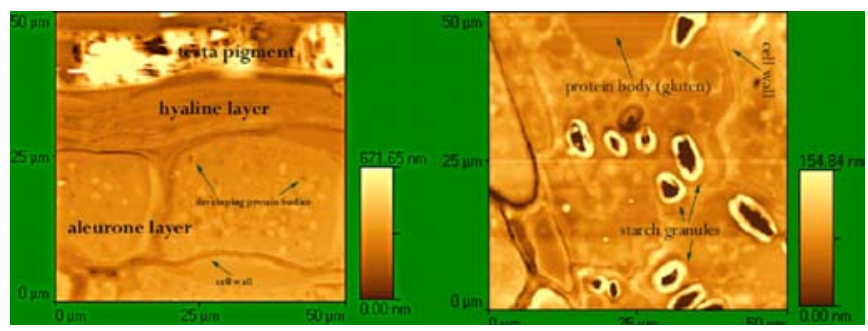

Figure 1 AFM image of wheat endosperm.

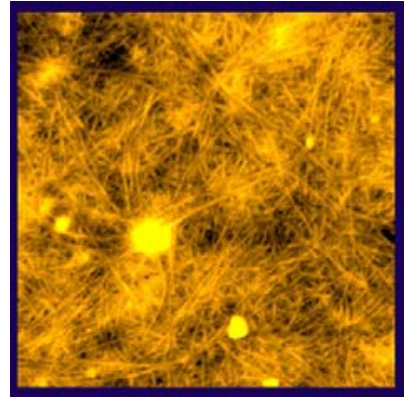

Figure 2 Surface of a polysaccharide gel at molecular resolution.

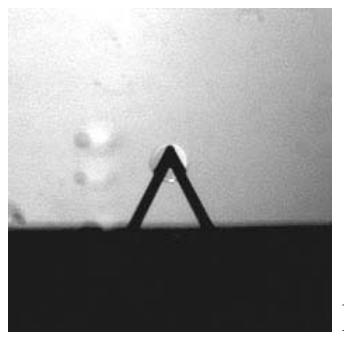

Figure 3 AFM cantilever with attached emulsion droplet. 